\title{
¿POR QUÉ USAMOS LAS TECNOLOGÍAS DE LA INFORMACIÓN Y LAS COMUNICACIONES? Un estudio sobre
} las bases sociales de la utilidad individual de Internet

\author{
WHY DO WE USE INFORMATION AND COMMUNICATION \\ TECHNOLOGIES? A Study on the Social Foundations of Internet \\ Individual Usefulness
}

Cristóbal Torres Albero cristobal.torres@uam.es

Universidad Autónoma de Madrid, España.

José Manuel Robles Morales jmrobles@ccee.ucm.es

Universidad Complutense de Madrid, España.

Óscar Molina Molina omolina@iesa.csic.es

IESA-CSIC, Córdoba, España.

\begin{abstract}
Resumen
Una de las cuestiones más controvertidas en el estudio de las Tecnologías de la Información y las Comunicaciones (TIC) es qué lleva a unos ciudadanos y no a otros a iniciarse en el uso de este tipo de tecnologías. La teoría más común en los estudios individualistas metodológicos sobre el uso de las TIC es la etiquetada como Technological Acceptance Model (TAM). Este modelo privilegia las variables individuales y subjetivas en la explicación del uso de Internet (percepción de utilidad y facilidad de uso de la tecnología). En nuestro trabajo aplicamos, a los datos de una encuesta nacional, una metodología estadística que incorpora al modelo TAM un conjunto de variables sociodemográficas. Con ello tratamos de mostrar en qué medida afectan estas últimas variables, fundamentalmente el nivel educativo y la edad, a las utilizadas por el TAM. Así, pretendemos ofrecer una versión sociológica del modelo TAM con una mayor capacidad explicativa.
\end{abstract}

\section{Palabras Clave}

Internet, Individualismo metodológico, Modelo TAM, Sociedad de la Información y el Conocimiento.

\section{Abstract}

One of the most controversial questions in the study of Information and Communication Technologies (ICT) is what leads some citizens and not others to start using this type of technology. The most common theory in individualist studies regarding the use of ICTs known as Technological Acceptance Model (TAM). This model prioritizes individual and subjective variables in explaining the study of Internet usage (perceived usefulness and perceived ease of use). In our paper we apply to the data from a national survey a statistical method that incorporates to the TAM model a set of social and demographic variables. Our aim is to show to what extent these variables, mainly level of education and age, affect the individual variables used by the TAM. Thus we aim to offer a sociological version of the TAM model that is characterized by its greater explanatory power.

\section{KEYWORDS}

Internet, Information and Knowledge Society, Methodological Individualism, TAM Model. 


\section{INTRODUCCIÓN ${ }^{1}$}

El surgimiento y desarrollo de un nuevo tipo de tecnología no supone, por sí mismo, un cambio en la forma de actuar, trabajar o pensar de los miembros de una sociedad (Mumford 1977). Gracias a estudios como De Gutenberg a Internet (Briggs y Burke 2002) hemos aprendido a relativizar el determinismo de las tecnologías y a comprender cómo diversas herramientas, nuevas y antiguas, pueden convivir en el mismo sistema. La transmisión de información y conocimiento oral no terminó con la aparición de la imprenta. La pintura, como forma de popularización de comportamientos y actitudes socialmente estimables o sancionables, no acabó, pese a todo, con la aparición de la fotografía. De la misma forma, la súbita aparición de las Tecnologías de la Información y las Comunicaciones (TIC), aun aceptando su incuestionable influencia, no han modificado por completo todos nuestros usos tecnológicos anteriores.

Denominamos "apropiación social de las tecnologías" al proceso mediante el cual una sociedad atribuye un determinado valor, una determinada utilidad o una determinada función a una tecnología (Echeverría 2008). Se trata de un proceso de larga duración en el que se ponen en juego los distintos intereses y recursos de los miembros del grupo que recepciona la tecnología. Desde este punto de vista, el impacto tecnológico depende, no sólo de la naturaleza de la tecnología en cuestión, sino también de la definición social que el grupo hace de dicha tecnología. Este concepto es, por lo tanto, de gran importancia para comprender cómo se usan las tecnologías disponibles en un momento histórico concreto, quién y con qué objetivos define sus usos y funciones, qué grupos sociales las aprecian y usan, qué tecnologías dejan de usarse o de tener un papel fundamental, en qué grado se produce esta sustitución, etc. Igualmente, es un concepto fundamental para entender el nivel de penetración de las tecnologías en la vida cotidiana de los individuos y, lo que tal vez sea más importante, los efectos que genera dicha incorporación en sus vidas.

Si seguimos a los autores de orientación crítica, entendemos este proceso de apropiación como un paso más en la evolución de la dominación social de aquellos con los recursos y las capacidades para definir el significado social de las tecnologías (Habermas 1984). De otro lado, si observamos esta secuencia desde un ángulo funcionalista nos llama la atención el papel de una u otra tecnología, por ejemplo en el desarrollo productivo u organizativo de una determinada comunidad (Drucker 1971). Por último, desde el ángulo constructivista se trata de responder a preguntas sobre los significados que los miembros de la comunidad conceden a esta tecnología, y sobre los efectos de dichas definiciones tienen en su entorno social (Knorr-Cetina 1999).

Pero el objetivo de este artículo es de índole más concreto y analítico. Se trata de estimar por qué algunos individuos y no otros comienzan a utilizar las TIC, y en particu-

\footnotetext{
${ }^{1}$ Este artículo ha sido posible gracias a los Proyectos de Investigación SEJ2006-12691, CSO2009-13771 y CSO 2009-13424, del Plan Nacional de Investigación I+D del Ministerio de Ciencia y Tecnología.
} 
lar Internet. Es decir, su fin es establecer qué determina que un ciudadano se decida a convertirse en internauta, si se debe a variables sociales como, por ejemplo, la edad, su nivel educativo o lugar de residencia 0 , por el contrario, hay que buscar las causas en un plano individual referido a la percepción que se tiene de la utilidad y la facilidad de uso de dicha tecnología. En definitiva, se trata de evaluar la pertinencia explicativa del enfoque individualista, de conocer sus límites y de valorar el papel que pueda tener una aproximación que conjugue estas variables y otras de carácter social.

Para atender a este objetivo el artículo se organiza en tres epígrafes principales. En el primero presentamos un estado de la cuestión de la teoría individualista con más predicamento entre la comunidad científica de este campo de estudio, la que conforma el Technological Acceptance Model (TAM). Esta breve revisión teórica nos permitirá mostrar que la principal línea de investigación en este marco teórico ya ha comenzado a girar su atención desde un enfoque fundamentalmente psicológico hacia explicaciones que incorporan variables de índole más social. En el apartado siguiente presentamos evidencia empírica a partir de una encuesta sobre el uso de las TIC en la población española. Sobre los resultados obtenidos hemos elaborado un modelo estadístico que nos ayudará a determinar el papel de las variables sociales y psicológicas en el uso individual de Internet. Los datos muestran que dicho uso depende, en mayor medida, de variables sociales (la edad y nivel educativo) que psicológicas. Con dichos resultados pretendemos aportar un caso de estudio que sea útil para el proceso de crítica y discusión del modelo TAM referido. En el último epígrafe interpretamos la evidencia empírica obtenida con la idea de desarrollar la tesis fundamental de este trabajo: el giro sociológico del TAM puede suponer un progreso en la capacidad explicativa de este modelo.

\section{El estado de la cuestión del Technological Acceptance Model}

Tal y como se ha apuntado en la Introducción, una de las cuestiones más controvertidas en el estudio de la difusión y desarrollo de las Tecnologías de la Información y las Comunicaciones es la de las razones qué llevan a unos ciudadanos y no otros a iniciarse en el uso de este tipo de tecnologías. Es decir, la cuestión sobre las causas y motivos que hacen que un ciudadano se convierta en internauta. Uno de los enfoques más habituales que han ofrecido las ciencias sociales apela a una explicación estructuralista. Así se ha estudiado, desde el ángulo de la "brecha digital", cómo determinadas variables sociodemográficas y socioeconómicas afectan al uso de tecnologías como Internet (Robles, Torres y Molina 2010). Sin embargo, existe también una larga tradición de estudios que abordan esta cuestión desde un ángulo individualista. Es decir, parten de un principio individualista metodológico (Elster 1996) para afrontar el estudio de las razones por las que los ciudadanos comienzan a utilizar las TIC.

En esta línea han primado los estudios de la psicología social (Davis 1986), pero no han faltado aportaciones de otras ciencias como la antropología, la economía o la propia sociología (Robles 2006). Este enfoque individualista ha servido, por otra parte, como 
marco teórico para un gran número de disciplinas aplicadas e interesadas en mejorar la penetración del uso de las TIC. Así, dicho enfoque ha inspirado el trabajo de especialistas en organización empresarial (Frambach y Schillewaert 2001), de técnicos de instituciones gubernamentales orientadas al desarrollo tecnológico (Cardoso, Mattos y Midori 2007) 0 de diseñadores de herramientas y dispositivos tecnológicos (Pavlou 2003). Por lo tanto, estamos ante una línea de trabajo interdisciplinar y aplicada, a la par que ante un marco teórico con un alto nivel de contrastación y aceptación empírica (Chau 1996).

Sin embargo, no es correcto hablar de una única teoría del comportamiento individual respecto al uso de las TIC. Para la explicación del uso de estas tecnologías se han aplicado varios constructos teóricos que, pese a tener gran número de elementos similares, distan en otros no menos importantes. Existen, al menos, siete teorías distintas utilizadas para el estudio teórico y empírico de este problema. Estas teorías son las siguientes: Theory of Reasoned Action (TRA), Technological Acceptance Model (TAM), Motivational Model (MM), Theory of Planned Behavior (TPB), Model of PC Utilization (MPCU), Innovation Diffusion Theory (IDT) y Social Cognitive Theory (SCT). Igualmente, existen dos teorías que tratan de aunar y conciliar las herramientas y conceptos de las siete anteriores. Éstas son la teoría unificadora del uso de las TIC (Venkatesh et al. 2003) y la versión extendida del TAM o TAM 2 (Szajna 1996). En este epígrafe nos referimos a la segunda de estas dos teorías, la TAM 2, puesto que el modelo unificado propuesto por Venkatesh y otros (2003) es más una reorganización de los principios del TAM 2 que un modelo sustantivamente diferente, mientras que la versión extendida del Technological Acceptance Model ha ido adaptando sus principios para acoger los elementos más importantes del resto de teorías anteriormente mencionadas.

De esta manera, presentando los conceptos y mecanismos del TAM 2 damos cuenta del estado de la cuestión en la explicación individualista del uso de las TIC que, en gran medida, ha corrido paralelo a la evolución del TAM (Thompson et al. 1991). Por otro lado, este marco teórico presta una atención especial a cuestiones contextuales y normativas. Nuestro enfoque, eminentemente sociológico, trata de avanzar justamente en esa dirección. Éstas son las razones por la que, dados los objetivos de este trabajo, hemos optado por este modelo en particular. Se trata, en definitiva, de tomarlo como base para la incorporación de algunos elementos explicativos de carácter sociológico.

El modelo original del Technological Aceptance Model se basaba fundamentalmente en dos conceptos básicos para la explicación del uso de las TIC: la Utilidad Percibida de dichas tecnologías y la Facilidad de Uso Percibida (Davis 1986; 1989). Cuando hablamos de Utilidad Percibida ${ }^{2}$ (UP) nos estamos refiriendo a una creencia subjetiva sobre las ventajas que esa tecnología puede tener para un individuo. Es decir, la probabilidad subjetiva de que el uso de determinada tecnología aumente el rendimiento del usuario 0 el provecho que obtiene de las tareas que realiza. Del mismo modo, la Facilidad de

\footnotetext{
${ }^{2}$ Todos los estudios empíricos de esta corriente concuerdan en que esta variable es el mejor predictor de la intención de utilizar las TIC (por ejemplo, Thomson et al. 1991).
} 
Uso Percibida (FUP) de una tecnología es una creencia sobre las facilidades que puede reportar esa tecnología para las actividades de un ciudadano en comparación con la tecnología utilizada con anterioridad ${ }^{3}$. EI TAM posiciona estos dos conceptos subjetivos en el centro de su modelo explicativo (Davis 1989).

Como complemento a los conceptos anteriormente mencionados, el TAM incorpora algunos aspectos fundamentales de la Theory of Reasoned Action ${ }^{4}$ (Davis et al. 1989). Dicha teoría, introducida por Fishbein y Ajzen en 1975, enriquecía la formulación básica del TAM con un concepto fundamental: la intencionalidad. En efecto, si UP y FUP facilitaban el estudio de los elementos subjetivos de la percepción individual de las tecnologías, la Theory of Reasoned Action (TRA) introduce el elemento objetivo y racional del modelo. En otras palabras, la TRA se centra en los elementos determinantes del comportamiento consciente de los individuos en este entorno concreto de decisión (Mathieson 1991). Para ello utiliza el concepto de Behavioral intention (BI). Según esta teoría el comportamiento concreto de una persona está determinado por su intención de realizar dicha acción (Mathieson 1991) ${ }^{5}$. A su vez, la intención se encuentra fuertemente influida por las actitudes de la persona hacia la tecnología, así como por las normas subjetivas relativas a la acción en cuestión (Davis et al. 1989). La actitud desempeña un papel fundamental en el modelo, ya que es el vínculo de unión entre los elementos subjetivos y los elementos intencionales. En otras palabras, la utilidad percibida y la facilidad de uso percibida de las tecnologías determinan, según la TAM, la actitud de los individuos respecto a dicha tecnología. Y dicha actitud está en la base de la intención de utilizarla. Sin embargo, el protagonismo otorgado a las actitudes por un modelo eminentemente individualista restó importancia a componentes de la TRA que recogían otros factores de carácter más contextual y normativo $0^{6}$. Pero si algo caracteriza al TAM 2 es un esfuerzo por incorporar esos y otros factores sociales y normativos en la explicación del comportamiento individual de los usuarios de las TIC (Szajna 1996).

Este esfuerzo tiene una de sus máximas expresiones en el trabajo de Malhotra y Galletta (1999). Estos autores introducen las ideas de Kelman (1958) sobre los cambios en las actitudes de los individuos como consecuencia de la influencia de determinados

\footnotetext{
${ }^{3}$ Las ideas que recogen estos dos conceptos han sido utilizadas en prácticamente todas las teorías de la aceptación de las TIC anteriormente enumeradas. Así, por ejemplo, la utilidad percibida es denominada en el Motivatinal Model como motivación extrínseca y como ventaja relativa en la Innovation Diffusion Theory. Por su parte, la facilidad de uso percibida se ha denominado expectativa de esfuerzo en el Modelo unificado y como complejidad en el Model of PC.

${ }^{4}$ La incorporación de los principios básicos de la Theory of Reasoned Action (TRA) supone otro ejemplo del carácter aglutinante del Technological Acceptance Model (TAM).

${ }^{5}$ Hasta aquí, simplemente se asevera que cualquier acción es una acción intencional. Ésta es una idea aceptada por la mayor parte de las teorías del comportamiento aunque no exenta de críticas. Para una discusión interesante, véase Searle (1980).

${ }^{6}$ Este es el caso, fundamentalmente, de las normas subjetivas que son un concepto muy importante en la explicación del comportamiento intencional propuesto en la TRA.
} 
factores externos. Estos factores son: conformidad, identificación e internalización. "Conformidad" se refiere al papel que tienen, de un lado, las sanciones y, de otro, los beneficios sociales para dar cuenta de un determinado comportamiento. En este caso, comenzar a utilizar una tecnología concreta. "Identificación" expresa la importancia de la sensación de pertenencia a un grupo y su influencia sobre la incorporación de comportamientos y actitudes propias de ese grupo. Por último, "Internalización" recoge las actitudes que incorporamos debido a su congruencia y consistencia con el resto de nuestros valores e ideas (Kelman 1958). Malhotra y Galletta (1999) denominan a la influencia conjunta de estos conceptos como lazos psicológicos (psycological attachment). Para ellos, estos elementos tenían un fuerte impacto sobre la actitud de los individuos respecto a las tecnologías, así como, directamente, sobre la intención de uso. El Esquema 1 representa las ideas expuestas hasta aquí, así como sus interrelaciones y dependencias.

Esquema 1.

Cuadro resumen TAM.

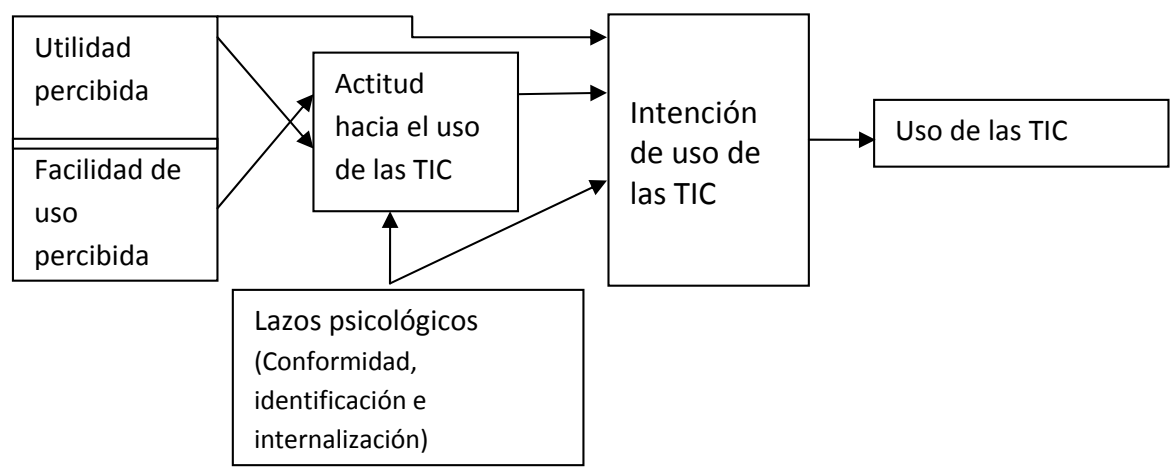

Fuente: Malhotra y Galletta (1999).

Hasta aquí hemos tratado de mostrar los conceptos básicos del modelo de explicación del uso de las TIC conocido como TAM. Hemos señalado que, para esta teoría, la intención de un agente individual de utilizar una determinada tecnología depende, fundamentalmente, de la actitud de este sujeto hacia la herramienta tecnológica. Dicha actitud es el resultado, a su vez, de tres tipos de factores: la utilidad percibida del uso de la tecnología, la facilidad de uso que el sujeto atribuye a dicha tecnología y los lazos psicológicos del individuo. El esquema básico de la TAM privilegia un enfoque psicológico e individual del uso de las tecnologías. Todas las variables contempladas en el modelo, se refieren a procesos mentales del agente. Esta afirmación también afecta a los lazos psicológicos, variables 
definidas por el modelo como contextuales ${ }^{7}$. Por lo tanto una persona determinada, usuaria potencial de Internet, es representada por la teoría como una agente que evalúa cuestiones como "la simpleza o complejidad del uso de Internet" y "las ventajas o desventajas que el uso de esta tecnología puede reportarle en comparación a otras tecnologías" de cara a definir su posición (actitud) tecnológica. Estos procesos psicológicos e individuales son los que finalmente explicarán la intención de la persona en cuestión de usar Internet.

Tal y como se ha señalado al inicio de este epígrafe, la capacidad explicativa de este modelo es bastante alta. En otras palabras, las variables definidas en TAM ayudan a explicar, en buena medida, qué impulsa a unos ciudadanos y no a otros a utilizar las TIC. Pero desde otro ángulo esta explicación es algo menos satisfactoria. Por ejemplo, apenas contamos con información sobre qué genera que un individuo considere más 0 menos útil una determinada tecnología. ¿Se trata de una evaluación individual estrictamente o está motivada por cuestiones que le vinculan con su entorno? Es decir, ¿la actitud hacia las TIC es dependiente, en alguna medida, del entorno? ¿La existencia de recursos e infraestructuras tecnológicas condiciona la actitud hacia las TIC de un individuo? ¿Afectan a su predisposición hacia la tecnología aspectos como la edad o los conocimientos que posee?

Como veremos más adelante, estas preguntas y otras similares han provocado un cierto desarrollo de lo social en el modelo TAM. Es lo que aquí venimos en llamar el giro sociológico del TAM que abordamos en un posterior punto. Pero antes, en el siguiente epígrafe, ofrecemos evidencia empírica para el caso de los ciudadanos españoles. En concreto, evaluamos qué tipo variables presentan una capacidad explicativa mayor respecto del uso de Internet: las sociológicas (edad, nivel de estudio, lugar de residencia, nivel económico o situación laboral) o las psicológicas propuestas por el modelo TAM que hasta aquí hemos referido. Nuestro objetivo es someter a la prueba empírica la solvencia del modelo, y comprobar hasta qué punto es necesario incluir variables sociológicas que mejoren la capacidad explicativa de esta teoría.

\section{EVIDENCIA EMPÍRICA DEL ESTUDIO DE CASO}

Presentamos en este epígrafe los resultados obtenidos a partir de una encuesta (UAM, 2008) estadísticamente representativa de la ciudadanía española, obtenidos mediante sistema CATI. La encuesta se llevó a cabo en el mes de noviembre de 2008 por la empresa Metroscopia, a partir de un diseño muestral representativo y polietápico estratificado con arreglo a las variables de comunidad autónoma y hábitat, y con cuotas representativas

\footnotetext{
${ }^{7}$ Consideramos que "los lazos psicológicos" es una variable psicológica más. Justificamos nuestra afirmación a partir de la dirección del proceso descrito por la teoría. No se trata de una influencia del contexto sobre la acción o la posición intencional del agente, sino de un proceso mental que le permite sentirse como parte de un grupo, y definirse a sí mismo a partir de unos determinados valores.
} 
en lo que respecta a las variables de sexo y edad. En total se entrevistaron a 1.000 personas de 18 años o más, lo que supone un margen de error estadístico máximo para los datos globales obtenidos del $\pm 3,1 \%$, asumiendo el supuesto más desfavorable $(P=Q=0,5)$ en la varianza o heterogeneidad poblacional, y con un nivel de confianza de 2 sigmas, es decir, con el 95,5\% de probabilidades de que la estimación de los datos muestrales obtenidos se ajuste a la realidad del conjunto del universo definido.

En un primer acercamiento descriptivo se observa que existe una fuerte relación entre las principales variables sociales y psicológicas y el uso de Internet, definido por haber utilizado esta tecnología en los últimos tres meses. Así, como apreciamos en el gráfico 1, cuanta más edad tienen los ciudadanos menor es el porcentaje de uso de Internet. Los ciudadanos mayores de 60 años que utilizan Internet apenas si alcanzan a un tercio de la población de individuos de esta edad. En el polo opuesto, los jóvenes (18-29 años) que utilizan esta tecnología superan el $95 \%$.

Gráfico 1.

Uso de Internet en España según edad.

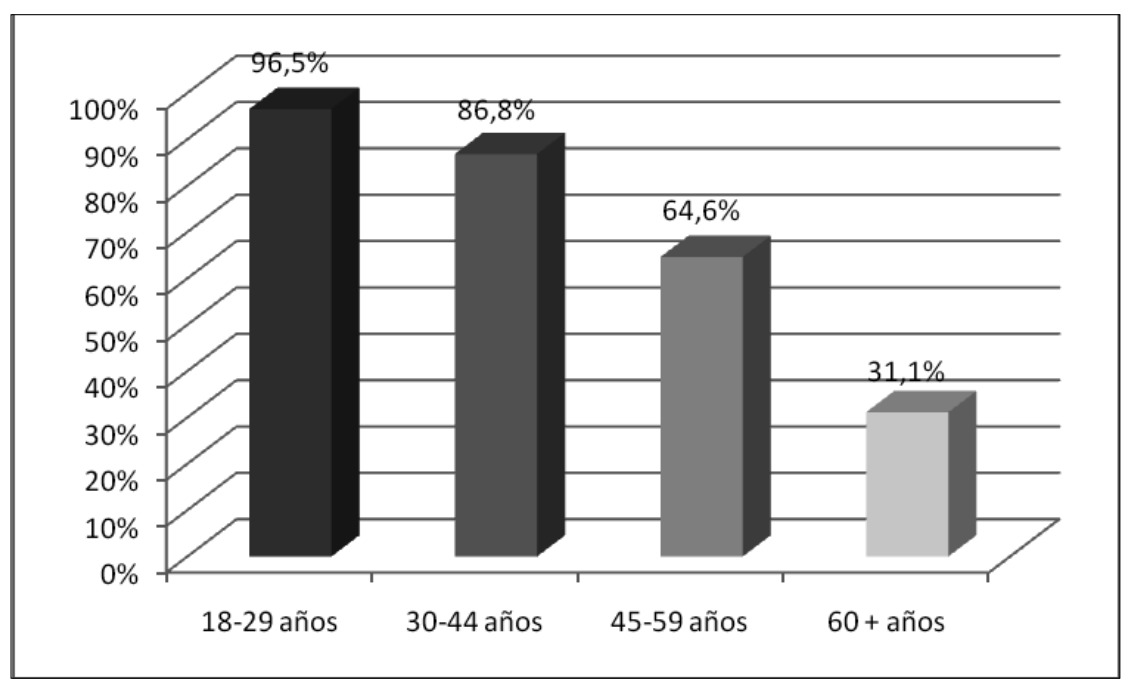

Fuente: Encuesta UAM 2008.

Esta circunstancia se repite en función del nivel educativo de los ciudadanos (gráfico 2). Una formación superior favorece la incorporación del uso de Internet a las actividades cotidianas de los ciudadanos. Nueve de cada diez universitarios son usuarios de Internet y más del $85 \%$ de los ciudadanos con estudios de secundaria utilizan esta tecnología. 
Sin embargo, este porcentaje desciende muy significativamente cuando nos referimos a personas que no cuentan con estudio básicos $(22,4 \%)$. De esta forma, podemos decir que la diferencia en la penetración de Internet entre personas muy formadas (universitarios) y personas sin formación, ronda los 70 puntos porcentuales.

Gráfico 2.

Uso de Internet en España según nivel educativo.

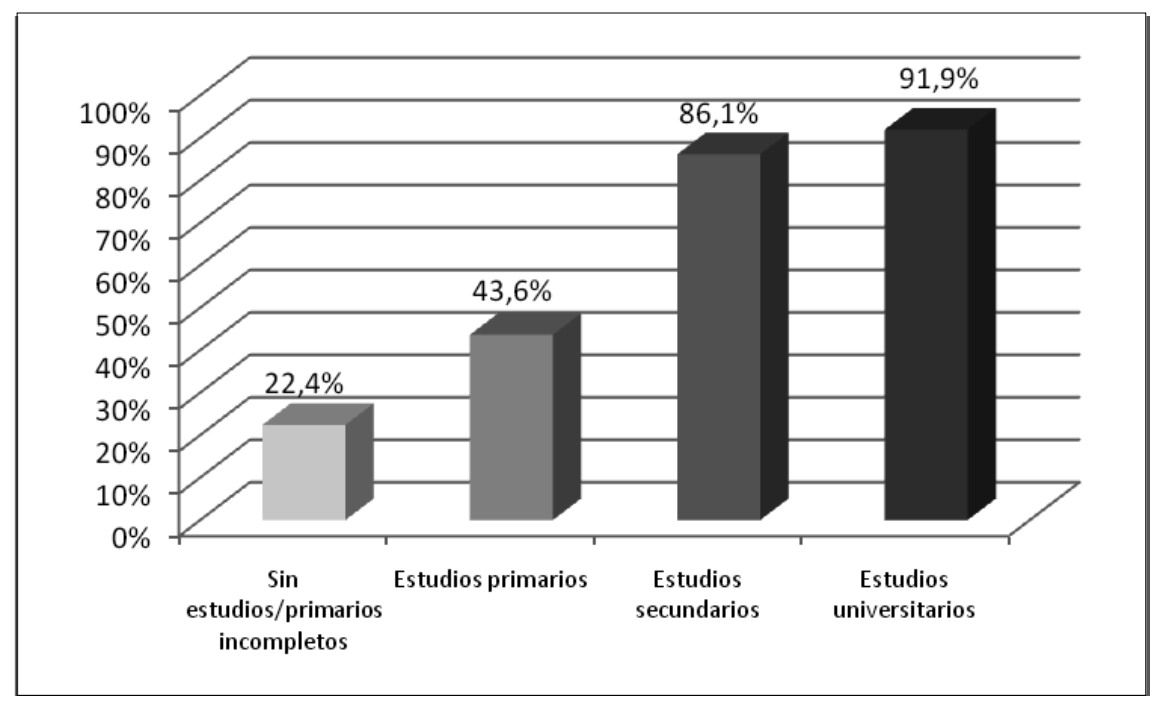

Fuente: Encuesta UAM 2008.

Pero las variables psicológicas también parecen afectar al uso de Internet. Las dos variables con más protagonismo en el TAM, la percepción de la utilidad de las TIC y la facilidad de uso de las TIC inciden, según nuestro estudio, en el uso de Internet de los españoles. En el gráfico 3 puede observarse que cuanto mayor es la percepción de la utilidad de esta tecnología, mayor es el uso de Internet. En nuestro estudio planteamos una escala numérica con un rango de entre 1 (mínima percepción de la utilidad) y 5 (máxima percepción de la utilidad). Así, los ciudadanos que más perciben la utilidad de Internet presentan un nivel de uso de esta tecnología del $90,1 \%$. De otro lado, los ciudadanos que no ven ninguna utilidad a Internet apenas hacen uso de ella (11,9\%). 
Gráfico 3.

Uso de Internet en España según la utilidad percibida del uso de esta tecnología.

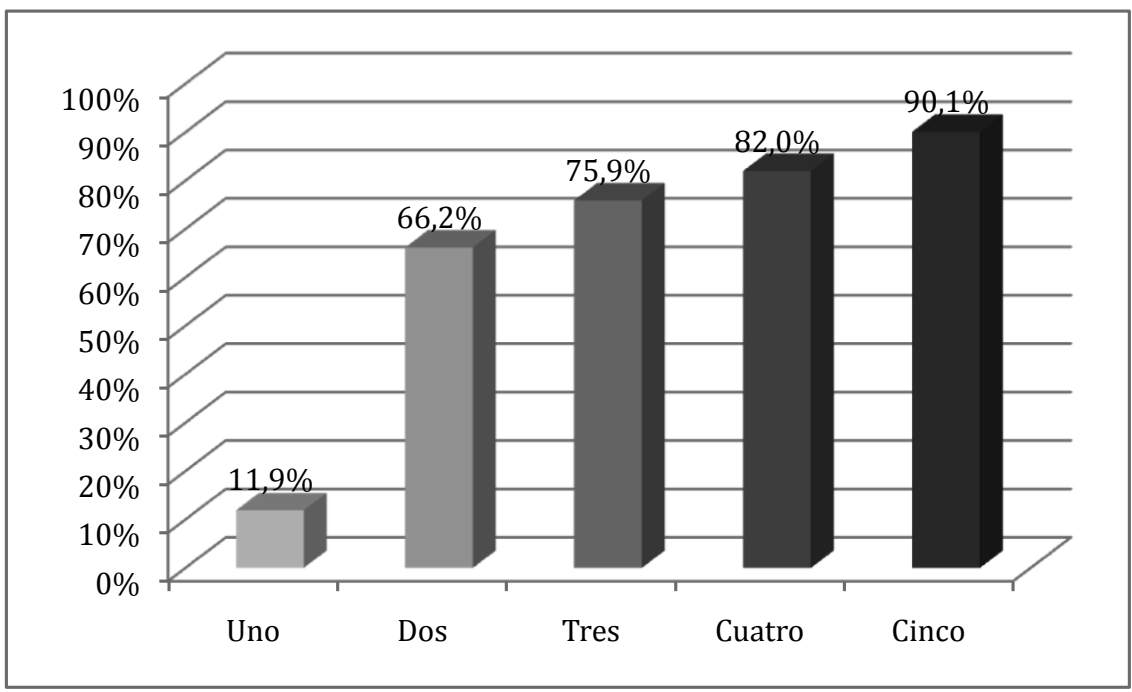

Fuente: Encuesta UAM 2008.

Por último, la facilidad percibida también afecta al uso de Internet (gráfico 4). El análisis de esta variable, con idéntica escala numérica, muestra que el $85 \%$ de los ciudadanos que perciben Internet como una tecnología sencilla de usar han incorporado a sus vidas dicha herramienta. Por el contrario, solo el $10 \%$ de los ciudadanos que expresan una dificultad máxima en el uso de Internet se declaran internautas.

En resumen, un primer acercamiento descriptivo a los datos empíricos del caso de estudio permite afirmar que tanto las variables sociales como las psicológicas parecen afectar al uso de Internet. Sin embargo, para un estudio más atento de esta relación necesitamos aplicar un método que evite el solapamiento y la injerencia de unas variables sobre otras, y nos permita cuantificar y ordenar el efecto de las variables sociales y psicológicas en el hecho de ser usuario o no de Internet. Tomando como referencia un análisis de este tipo puede avanzarse en la respuesta al objetivo formulado en este artículo.

Para este fin el método seleccionado ha sido el de la regresión logística. Más concretamente, su modalidad "condicional hacia delante". Hemos tomado como variables independientes las variables sociodemográficas habituales (nivel de estudios, edad, sexo, clase social, situación laboral, tamaño de hábitat e ideología) y las variables psicológicas propuestas por el TAM (utilidad y facilidad percibida). La variable dependiente es la de haber usado Internet en los últimos tres meses. Los resultados obtenidos per- 
Gráfico 4.

Uso de Internet en España según percepción de facilidad de esta tecnología.

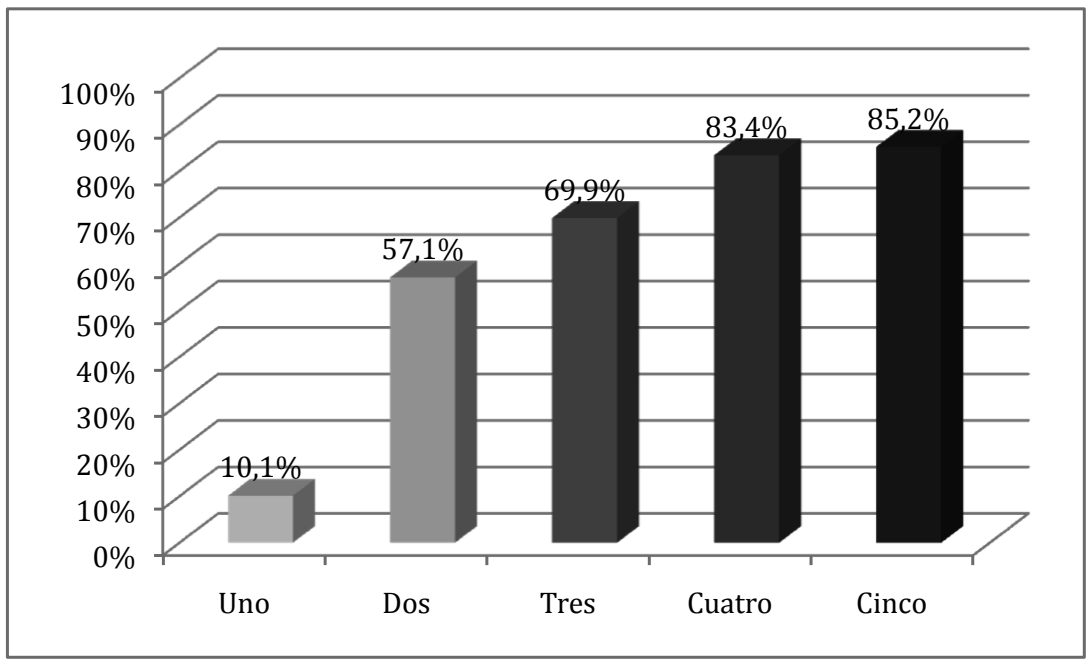

Fuente: Encuesta UAM 2008.

miten afirmar, en primer lugar, que las variables consideradas, excepto el tipo de hábitat y la situación laboral, son significativas para explicar el uso individual de Internet. En segundo lugar, también puede indicarse que el ajuste del modelo es aceptable, con un $\mathrm{R}$ cuadrado de Nagelkerke igual a 0,672 , y el $86,8 \%$ de los casos clasificados correctamente. En definitiva, existe una alta correlación entre la variable dependiente y las variables independientes seleccionadas que han resultado significativas.

A partir de esta base nos preguntamos por el grado de influencia que las diferentes variables independientes identificadas tienen para dar cuenta del uso de Internet. Existen varios procedimientos cuyo fin es la estandarización de los coeficientes obtenidos en los modelos de regresión logística, y así poder compararlos y establecer una jerarquía u orden de las variables que determinan la variable dependiente. En nuestro caso todas las variables explicativas son categóricas, por lo que se ha obtenido un coeficiente para cada categoría distinta de la de referencia inicial. Estos coeficientes nos permiten determinar las comparaciones más relevantes, pero no nos permiten saber las variables que influyen en mayor o menor medida sobre el uso de Internet. Existen otras formas de valorar el aporte explicativo de las variables en un modelo de regresión logística, ya sea a partir de determinados estadísticos (como por ejemplo, el estadístico de Wald) o de conceptos como la "verosimilitud" y la "lejanía". 
La verosimilitud es una medida que se calcula a partir de los datos de la muestra y se utiliza para valorar el grado en que el modelo arroja resultados coherentes con la condición que realmente tienen los sujetos. Si llamamos $\mathrm{Pa}$ la probabilidad estimada por el modelo de que un individuo de la muestra sea usuario de Internet, sería deseable que el modelo asignara probabilidades altas (cercanas a 1) a los sujetos que realmente son usuarios de Internet y probabilidades bajas (cercanas a 0) a los no usuarios (es decir, probabilidades altas P'=1-P de no usar Internet). El producto de todas las probabilidades, P para usuarios y P' para no usuarios, es conocido como verosimilitud del modelo (se representa como V). La proximidad de la verosimilitud a 1 indica que el modelo es eficiente para modelar la realidad, mientras que una verosimilitud cercana a 0 revela que el modelo no es idóneo para nuestro propósito.

Debido a que la verosimilitud mide la plausibilidad de un modelo de regresión logística, es central considerarla cuando tratamos de medir su capacidad predictiva. No obstante, lo que se suele utilizar es una transformación de la verosimilitud $(L=-2 \ln V)$ que se conoce con el nombre de lejanía del modelo. Como $V$ varía entre 0 y 1 , su logaritmo siempre será negativo, de modo que la lejanía siempre será un número positivo. Cuanto más eficiente sea un modelo, mayor será su verosimilitud (más próxima a 1) y, por tanto, menor su lejanía.

Partiendo de estos conceptos de verosimilitud y lejanía, vamos a valorar el aporte explicativo de cada variable incluida en el modelo. No debe sorprendernos que el modelo nulo (sin variables explicativas, sólo con el término constante) sea el que mayor lejanía presenta, y que a medida que se incluyen variables en el modelo la lejanía va disminuyendo paulatinamente. Estas disminuciones que experimenta la lejanía cuando se incluyen variables en el modelo son proporcionales al aporte explicativo de las variables, de forma que si una variable explica una parte considerable de la variable dependiente no explicada por el resto de variables independientes, la lejanía se reducirá de forma notable. Análogamente, cuando el aporte explicativo es pequeño también será escasa la reducción de la lejanía.

Para analizar y cuantificar el aporte explicativo de cada variable incluida en el modelo hemos considerado dos lejanías: la del modelo con todas las variables explicativas que han resultado ser significativas; $y$ la del modelo con todas las variables explicativas salvo la variable que estamos examinando. La diferencia entre ambas lejanías nos indica la contribución de cada variable a la explicación del uso de Internet. Estas diferencias se pueden hacer relativas respecto a la lejanía del modelo con todas las variables, obteniendo de esta forma el porcentaje (\%) de aumento de la lejanía al eliminar cada una de las variables significativas del modelo elaborado. Los resultados obtenidos se presentan en la tabla 1.

Como se puede apreciar en la tabla 1 , la edad es la variable con un mayor aporte explicativo, aumentando la lejanía del modelo un $27,5 \%$ si se elimina. Le sigue, con porcentajes explicativos notablemente inferiores, el peso explicativo del nivel educativo $(10,6 \%)$, la utilidad percibida de Internet $(6,1 \%)$ y la facilidad de uso percibida $(5,4 \%)$. Las variables de sexo $(0,9 \%)$, clase social $(1,2 \%)$ e ideología $(2,5 \%)$ son las que menos 
Tabla 1.

Modelo si se elimina la variable

SEXO

EDAD

NIVEL DE ESTUDIOS

CLASE SOCIAL

IDEOLOGÍA

UTILIDAD PERCIBIDA

FACILIDAD DE USO PERCIBIDA
$\Delta R^{2}$ : cambio en $-2 \ln V$

$0,9 \%$

$27,5 \%$

$10,6 \%$

$1,2 \%$

$2,5 \%$

$6,1 \%$

$5,4 \%$

Nota: Los porcentajes que aparecen en la tabla indican la pérdida de poder explicativo del modelo al eliminar la variable en cuestión. No entran en el modelo las variables, por no ser significativas, del tipo de hábitat y la situación laboral.

Fuente: Encuesta UAM 2008

contribuyen a la explicación de la variable dependiente, pues la lejanía del modelo apenas aumenta cuando se eliminan.

Debido a que no siempre la significatividad y la importancia son lo mismo, especialmente en muestras muy grandes o muy pequeñas, decidimos repetir el modelo de regresión logística sin incluir las variables de sexo, clase social e ideología, pues si bien resultan significativas, su aporte explicativo es muy escaso. El ajuste del nuevo modelo que se obtiene, y que se presenta en la tabla 2, es muy similar al del modelo anterior, a excepción del nivel educativo, con una aportación explicativa bastante superior $(21,3 \%)$, similar al que supone la variable edad $(24,0 \%)$. Esta mayor relevancia del nivel educativo, que es consistente con los datos obtenidos en un estudio anterior que realizamos para explicar la brecha digital en España (Robles, Torres y Molina 2010), se debe al hecho de haber dejado fuera del segundo modelo variables que comparten gran parte de información explicativa con el nivel educativo. En concreto la pertenencia a una clase social u otra está muy relacionada con el nivel educativo, puesto que viene definida por el cruce de situación laboral y nivel educativo, por lo que gran parte de lo explicado por ambas variables sobre el uso de Internet es común.

Una vez obtenidos estos resultados podemos afirmar, para el caso español, que las variables sociales de la edad y el nivel de estudios tienen una gran influencia sobre el uso de Internet entre los españoles. Tanto es así que superan sensiblemente el peso

\footnotetext{
${ }^{8}$ Las encuestas utilizadas en este y en aquel estudios no son coincidentes. Tanto el método de encuestación como la muestra son diferentes. Pese a ello, es importante destacar que los resultados de aquel análisis y los mostrados en este estudio nos permiten llegar a conclusiones similares.
} 
Tabla 2.

\begin{tabular}{ll}
\hline Modelo si se elimina la variable & $\Delta \mathrm{R}^{2}$ : cambio en $-2 \operatorname{lnV}$ \\
\cline { 2 - 2 } EDAD & $24,0 \%$ \\
NIVEL DE ESTUDIOS & $21,3 \%$ \\
UTILIDAD PERCIBIDA & $5,9 \%$ \\
FACILIDAD DE USO PERCIBIDA & $5,4 \%$
\end{tabular}

Nota: Los porcentajes que aparecen en la tabla indican la pérdida de poder explicativo del modelo al eliminar la variable en cuestión. No entran en el modelo las variables del tipo del hábitat, situación laboral, sexo, clase social e ideología.

Fuente: Encuesta UAM 2008.

que ejercen las variables psicológicas de utilidad y facilidad percibida propuestas por el TAM. Ello nos lleva, en el siguiente apartado, a considerar con más detalle el desarrollo que el modelo TAM ha tomado a partir de la consideración que ha hecho de las variables sociales. Nuestro objetivo es mediar en este debate y proponer lo que venimos en llamar un giro sociológico del TAM.

\section{EL GIRO SOCIOLÓGICO DEL TAM}

Tal y como mencionamos anteriormente, uno de los campos más fructíferos de desarrollo del TAM a lo largo de los últimos años ha sido el estudio de la influencia de los factores sociales, normativos y contextuales sobre el uso de las TIC, y especialmente de Internet. A partir del modelo original, diversos estudios han sofisticado y mejorado la capacidad explicativa recurriendo a variables sociales.

Una de las líneas más interesantes se refiere al papel que desempeñan las condiciones materiales de acceso a las TIC sobre la actitud tecnológica de los individuos. EI papel de las infraestructuras de acceso a las TIC ha sido uno de los elementos que el TAM ha incorporado de la Innovation Difussion Theory (IDT). Con ello, se buscaba analizar en qué medida la percepción subjetiva de la suficiencia e idoneidad de las condiciones materiales y recursos disponibles influyen en las intenciones de los individuos. Es decir, se trata de estimar lo que se considera como "condiciones de facilitación" del uso de las TIC, definidas como el grado en el cual un individuo considera que existen infraestructuras tecnológicas que posibilitan el uso de las TIC (Taylor y Todd 1995). De esta forma, junto a las tres variables clásicas del modelo referido, utilidad percibida, facilidad de uso y lazos psicológicos, se ha incorporado un concepto que analiza la influencia de la percepción subjetiva de las infraestructuras existentes en un determinado territorio sobre la actitud de los usuarios potenciales de las TIC. 
Otro de los trabajos más influyentes en el estudio de la relación entre contexto y uso de las TIC es el realizado por Venkatesh et al. (2003). Estos autores investigan cómo influyen las variables sociales de la edad, género, experiencia y voluntariedad sobre la actitud hacia las tecnologías. El interés de los autores por el papel de las dos últimas variables referidas se debe a la naturaleza organizativa y empresarial de sus trabajos. Aquí, dado nuestro objetivo, sólo haremos mención a la influencia de las variables de edad y sexo. Su estudio es una muestra representativa de un gran número de investigaciones que analizan el destacado papel que juegan el sexo y la edad sobre la percepción de la utilidad de las tecnologías y la facilidad de uso de las mismas. En esta línea, Lynott y McCandless (2000) han mostrado el fuerte impacto que tiene el género sobre la percepción de la utilidad de las TIC. Así, los hombres presentan una predisposición o una actitud más favorable hacia el uso de tecnologías como Internet debido a que perciben, en mayor medida que las mujeres, la utilidad de estas tecnologías. Este hecho se debe, según estos últimos autores, más a una cuestión de socialización y aprendizaje que a una cuestión estrictamente relacionada con la naturaleza del género. De otro lado, la edad es otro factor que influye sobre la percepción de la utilidad de las tecnologías (Morris y Venkatesh 2000). Lo mismo puede decirse de la facilidad percibida de uso, puesto que diversos estudios muestran la fuerte influencia entre las variables edad y género y lo fácil o difícil que los individuos perciben el uso de las tecnologías (Plude y Hoyer 1995).

Dados estos precedentes, podría pensarse que uno de los debates más prósperos abiertos por el modelo TAM en el estudio del uso de las TIC en general, y de Internet en particular, tendría que ver con el papel explicativo de las variables sociales sobre esta conducta individual. Pero a pesar de estos avances, y de la previsible fertilidad explicativa que pueden alcanzar las variables sociales, en nuestra investigación no hemos encontrado estudios recientes que incorporen al análisis otras variables sociodemográficas distintas a las mencionadas. No desechamos la posibilidad de que existan estudios orientados en esta dirección. Pero en tal caso, su impacto y trascendencia es aún limitado habida cuenta de que no quedan recogidos en el gran número de estudios analizados en nuestro trabajo, y que aparecen en la bibliografía.

Por ello, consideramos que aún restan muchas cuestiones por analizar en una línea de trabajo que subraye el papel de las variables sociales. Tal y como se mencionó más arriba, la utilidad percibida es la variable que ha demostrado más influencia sobre el uso de las TIC desde la perspectiva TAM. Pero la introducción de variables sociales abre interrogantes sobre la validez de esta fuerte influencia. Así, en primer lugar, sería primordial identificar cuáles de las variables sociales explican en mayor medida el uso individual de las TIC. Por ejemplo, es importante ampliar nuestro conocimiento sobre el papel de otras variables sociales tan relevantes como el nivel educativo o el lugar de residencia del individuo (Robles, Torres y Molina 2010). Sobre esta base, y en segundo lugar, se plantea la cuestión de si continúan siendo las variables psicológicas las que más explican el uso de las TIC. Por último, y como conclusión, podríamos preguntarnos por el papel conjunto de las variables psicológicas y sociales en la explicación del uso de Internet. 
El diseño de nuestro estudio empírico se realizó teniendo como horizonte dar respuesta a estas y otras cuestiones similares. Así, la metodología utilizada nos permite ofrecer información sobre la influencia relativa que ejerce cada una de las variables independientes recogidas en el modelo sobre la variable dependiente (uso de Internet). De esta manera hemos registrado cómo, en un primer momento, la edad se transforma en la variable con mayor incidencia sobre el uso de Internet. En la primera prueba, en la que recogemos todas las variables contempladas, la pérdida de capacidad explicativa del modelo cuando no se toma en cuenta la edad es de un 27,5\%. Este resultado apoyaría la tesis expuesta en alguno de los estudios, ya mencionados, como los de Morris y Venkatesh (2000) y Venkatesh et al. (2003). Es decir, el resultado de nuestro estudio estaría mediando en el actual estado de la cuestión al proporcionar información que corrobora los estudios iniciados por estos autores para el caso concreto de España.

Sin embargo, la segunda de las formulaciones expuesta en el apartado empírico de nuestro trabajo nos permite dar un paso más en la investigación sobre el papel de las variables sociales en el estudio del comportamiento tecnológico descrito por la TAM. En este segundo modelo, donde sólo se recogen las variables con mayor incidencia, la que tiene una mayor capacidad explicativa continúa siendo la edad, pero el nivel educativo adquiere una importancia mucho mayor. En este caso, la eliminación de esta variable implicaría la pérdida de un $21,3 \%$ de capacidad explicativa del modelo. Este resultado tiene una gran importancia para el giro sociológico de la TAM ya que es, la primera vez, que la formación del individuo es introducida como una variable explicativa en el estudio del uso de Internet. En otras palabras, tras la apertura sociológica del TAM con variables como la edad o el género, nuestra principal aportación a este debate es la inclusión de esta tercera variable social a la lista contemplada por la literatura.

Al hilo de lo anterior es importante señalar cómo la variable sexo apenas presenta, en nuestro modelo, impacto sobre el uso de Internet. Es decir, de no contar con esta variable el modelo explicativo utilizado perdería menos de un $1 \%$ de su capacidad explicativa. Así pues, nuestro estudio no coincide en este aspecto con los resultados apuntados por la literatura que inauguró el giro sociológico del TAM. Consideramos que este hecho se debe, en gran medida, a que las variables edad y nivel educativo subsumen la información explicativa que anteriormente generaba la variable género.

Otra de las conclusiones que pueden extraerse del estudio empírico tiene que ver con la diferente capacidad explicativa de las variables sociales y psicológicas. Tanto en la aplicación en la que contemplamos todas las variables independientes, como en la que utilizamos sólo aquellas que más influencia presentan sobre el uso de Internet (edad, nivel de estudio, percepción de la utilidad y de la facilidad de uso de Internet) la influencia de las variables sociales es sensiblemente superior a la de las variables psicológicas. Es decir, si elimináramos la variable "edad", el modelo perdería una capacidad explicativa del $24 \%$. Por otra parte, al sacar de este modelo la variable del "nivel educativo" nuestra capacidad de explicar el uso de Internet se reduciría en un 21,3\%. Por el contrario, la pérdida de capacidad explicativa sería mucho menor en el caso de no contar con las dos principales variables descritas por el modelo TAM: la utilidad y 
facilidad de uso percibida. Eliminando la variable "utilidad percibida", perderíamos el $5,9 \%$ de la capacidad explicativa de nuestro modelo. Mientras, si prescindiéramos de la "facilidad percibida" la pérdida sería de $5,4 \%$. Por tanto, de nuestro estudio del caso español puede extraerse la conclusión de la preponderancia de la capacidad explicativa de las variables sociales sobre las variables psicológicas.

Pero más allá de estas consideraciones cuantitativas, este resultado nos permite reflexionar acerca del significado que tiene que la edad y el nivel de estudio sean las variables que presenten mayor incidencia sobre el uso de Internet. Desde nuestro punto de vista, la edad hace referencia principalmente a la socialización tecnológica de los ciudadanos. Los jóvenes han crecido en un entorno en el que las TIC forman parte fundamental de sus vidas. Como es sabido, la sociedad de la información y del conocimiento ha arrancado en los últimos veinticinco años, por lo que puede decirse que los jóvenes son el único grupo social cuya socialización se ha producido enteramente en presencia de estas tecnologías. La adquisición de las pautas de uso de las TIC, como de cualquier otro artefacto vinculado a la acción social, supone una combinación de conocimiento y aprendizaje formal de un lado y, de otro, de habilidades y experiencias. Pero además la socialización funciona como un troquelado que actúa sobre las disposiciones físicas y culturales de nuestra vida. Por ello, cuanta más edad tiene una persona más difícil es eliminar el modelado que nos ha generado la socialización primaria y secundaria y, de otra, más arduo es adquirir el nuevo conocimiento y las pericias necesarias para usarlo. Así pues los jóvenes disponen de más ventaja, en términos comparativos con respecto al resto de cohortes poblacionales, en el aprendizaje e interiorización de cualquier pauta 0 artefacto novedoso vinculado a las TIC (Torres 2009).

En esa línea es posible entender por qué el uso diferencial de las TIC no sólo es privativo de los jóvenes sino también, como nos indicaban los datos del INE ${ }^{9}$, de los niños. No es ajeno a este fenómeno que uno de los rasgos actuales de las sociedades avanzadas es la progresiva rebaja de la edad de incorporación de los niños al mundo juvenil, con lo que supone de la adquisición a una edad más temprana de las habilidades, usos e identidades propias de la juventud.

Una reflexión parecida puede hacerse al hablar del papel del nivel educativo en el uso de Internet. Si existe una idea que comparten los distintos expertos sobre las consecuencias sociales de la sociedad de la información y del conocimiento es que la adquisición de la capacidad intelectual para obtener información digital, saber recombinarla y utilizarla para producir conocimientos es un referente obligatorio que introduce la nueva sociedad informacional. Algo que, sobre todo, puede interiorizarse especialmente en las instituciones educativas a lo largo de la socialización secundaria. De otro lado, el nivel

\footnotetext{
${ }^{9}$ La encuesta de 2009 del INE sobre Equipamiento y uso de TIC en los Hogares indica que el 85,1\% de los niños de 10 a 15 años han accedido a Internet en alguna ocasión en los últimos tres meses. Los datos detallados, así como la evolución desde 2004 a 2009, puede consultarse en http://www.ine.es/inebmenu/ mnu_tic.htm\#2
} 
de estudios de los ciudadanos está muy relacionado con el entorno familiar y cultural de los individuos. Adicionalmente, existe también consenso en que la educación y el aprendizaje a lo largo de toda la vida de las personas constituyen herramientas esenciales para el trabajo y el desarrollo personal en este nuevo tipo de sociedad (Castells 2001). Quienes han incorporado las TIC desde edad más temprana, y presentan una mayor ventaja educativa, tienen mayores probabilidades de ventajas para afrontar este reto.

En definitiva, la educación no sólo hace referencia a cuestiones que tienen que ver con la formación formal de los ciudadanos, sino que esta enlazada con una amplia gama de factores que tratan de explicar el desarrollo educativo de los ciudadanos a partir de otros vectores como la edad o el entorno familiar y cultural. Así pues, al igual que la variable edad, el nivel de estudios es una forma de estudiar la vinculación entre el individuo y lo social. Mostrar, tal y como hemos hecho aquí, que la educación de los ciudadanos influye sobre el uso de Internet, tiene implicaciones psicológicas más generales y explicativas que las alcanzadas a partir del análisis aislado de las preferencias individuales.

\section{Conclusiones}

En este artículo no hemos perseguido poner en cuestión la validez de la aproximación psicológica que supone el modelo TAM. Supondría retroceder a épocas muy pretéritas de la teoría sociológica el enfrentar de manera excluyente el enfoque individual que implica la psicología con la aproximación estructural que habitualmente se asocia con la sociología. El objetivo de este trabajo ha sido mostrar la importancia de aplicar una estrategia multidisciplinar para un fenómeno tan complejo como la adopción de las tecnologías.

EI TAM, un modelo eminentemente psicológico e individualista, ha sido un enfoque pionero y riguroso en la explicación del uso de las TIC, y de Internet en particular. Aunque ha desarrollado una cierta orientación hacia lo social, incorporando variables que permitan comprender cómo se vinculan con el entorno los individuos estudiados, a nuestro juicio dicha aproximación ha sido bien insuficiente para una perspectiva sociológica. Por ello, hemos apostado por subrayar la importancia de una estrategia que desarrolle lo que hemos calificado como el giro sociológico del TAM.

Los resultados de nuestro caso de estudio avalan sobremanera la necesidad de esta reorientación, tanto porque corroboran algunos de los estudios desarrollados con disposición social bajo el modelo TAM (la importancia de la edad), como porque introducen y destacan, por primera vez, la importancia del nivel educativo para explicar el uso de Internet. La centralidad de los factores sociales también queda expresada en el significativo mayor peso explicativo que éstos tienen sobre las variables psicológicas cruciales del referido modelo (percepción de utilidad y facilidad de uso). 
En definitiva, los resultados del estudio de caso que hemos acometido indican que la formulación del TAM podría potenciar su capacidad explicativa incorporando variables que permitan comprender cómo se vinculan socialmente los individuos estudiados. Así pues, el futuro de nuestro trabajo, en la siempre densa agenda investigadora de la sociedad de la información y del conocimiento, pasa por el desarrollo de un modelo alternativo sobre el proceso de apropiación de Internet más orientado hacia una línea de explicación netamente sociológica que, no obstante, debe aprovechar el camino teorético y empírico ya avanzado por el TAM en su aproximación individual y psicológica.

\section{REFERENCIAS BibLIOGRÁFICAS}

Cardoso, G., I. Mattos y C. Midori. 2007. "Modeling the acceptance and de use of telecenters in Brazil." 2:86-97.

Castells, M. 2001. La galaxia Internet. Barcelona: Areté.

Chau, P. Y. K. 1996. "An empirical assessment of a modified technological acceptance model." Journal of Management Information Systems 13:185-204.

Briggs, A. y P. Burke. 2002. De Gutenberg a Internet. Madrid: Taurus.

Davis, F. D. 1986. A Technological Acceptance Model for empirical testing new end-user information systems: Theory and results. Cambridge: MIT Sloan School of Management.

Davis, F. D. 1989. "Perceived usefulness, perceived ease of use and user acceptance of information technology." MIS Quarterly 13:319-340.

Davis, F. D., R. P. Bagozzi y P. R. Warshaw. 1989. "User acceptance of computer technology: A comparison of two theoretical models." Management Science 35:982-1002.

Drucker, P. F. 1971. La gran ruptura; perspectivas socioeconómicas para el mundo del futuro. Buenos Aires: Troquel.

Echeverría, J. 2008. "Apropiación social de las tecnologías de la información y la comunicación." Revista iberoamericana de ciencia, tecnología y sociedad 4:171-182.

Elster, J. 1996. Tuercas y Tornillos. Una Introducción a los conceptos básicos de las Ciencias Sociales. Barcelona: Gedisa.

Frambach, R. T. y N. Schillewaert. 2001. "Organizational Innovation adoption: a multi-level framework of determinants and opportunities for future research." Journal of Business Research 55:163-176.

Fishbein, M. e I. Ajzen. 1975. Belief, attitude, intention and behavior: and introduction to theory and research. Nueva York: Addison-Wesley.

Habermas, J. 1984. Ciencia y técnica como ideología. Madrid: Tecnos. 
Kelman, H. C. 1958. "Compliance, identification and internalization: Three processes of attitude change?." Journal of Conflict Resolution 2:51-60.

Knorr-Cetina, K. D. 1999. Epistemic cultures. How science make knowledge. Londres: Havard University Press.

Lynott, P. P. y N. J. Mccandless. 2000. "The impact of age vs. life experiences on the gender role attitude of women in different cohorts." Journal of Women 12:5-21.

Malhotra, Y. y D. F. Galletta. 1999. "Extending the Technology Acceptance Model to Account for Social Influence: Theoretical Bases and Empirical Validation." Documento de trabajo para la 32 edición del encuentro sobre sistemas científicos celebrado en Hawai en 1999.

Mathieson, K. 1991. "Predicting user intentions: comparing the technological acceptance model with the theory of planned behavior." Information Systems Research 2:173-191.

Morris, M. G. y V. Venkatesh. 2000. "Age differences in technology adoption decisions." Personnel Psychology 53:375-403.

Mumford, L. 1997. Técnica y civilización. Madrid: Alianza.

Pavlou, P. A. 2003. "Consumer acceptance of electronic commerce: integrating task and risk with the technological acceptance model." International Journal of Electronic Commerce 7:101-134.

Plude, D. J. y W. J. Hoyer. 1995. "Age and selectivity of visual information processing." Psychology and Aging 7:133-145.

Searle, J. 1980. "The intentionality of intention and action." Cognitive Science 4:47-70.

Szajna, B. 1996. "Empirical evaluation of the revised technology acceptance model." Management Science 42:85-99.

Robles, J. M. 2006. "Las creencias cuentan: el papel de la percepción de la utilidad de las TIC en el desarrollo de la Sociedad de la Información". Pp. 215-228 en TIC y Sociedad en el Siglo XXI, editado por D. Becerril. Granada: Universidad de Granada.

Robles, J. M., C. Torres y O. Molina. 2010. "Las fuentes de las desigualdades tecnológicas en España. Un estudio sobre las nuevas formas de desigualdad social." Sistema 218: 3-22.

Taylor, S. y P. A. Todd. 1995. "Understanding information technology usage: A test of competing models." Information Systems Research 6:144-176.

Thompson, T., C. A. Higgins y J. M. Howell. 1991. "Personal computing. Toward a conceptual model of utilization." MIS Quarterly 15:125-143.

Torres Albero, C. 2009. "Jóvenes y TIC: una afinidad electiva". Pp: 471-494 en Juventud y exclusión social, editado por J. F. Tezanos. Madrid: Editorial Sistema.

UAM 2008. Encuesta sobre representaciones sociales de la Ciencia y Tecnología. Universidad Autonoma de Madrid: Madrid.

Venkatesh, V., M. G. Morris, G. B. Davis y F. D. Davis. 2003. "User acceptance of information technology: Toward a unified view", MIS Quarterly 27:425-478. 
CRISTÓBAL TORRES ALBERO es Catedrático de universidad en el Departamento de Sociología de la Universidad Autónoma de Madrid. Su campo de trabajo prioritario es la sociología de la ciencia y la tecnología así como los estudios sociales de la sociedad de la información y del conocimiento.

JOSÉ MANUEL ROBLES MORALES es Profesor Titular (interino) de la Sección departamental de Sociología III en la Facultad de Ciencias Económicas y Empresariales de la Universidad Complutense de Madrid. Está interesado en la relación entre tecnología, sociedad y política y es especialista en participación política, acción colectiva, democracia digital y brecha digital.

ÓSCAR MOLINA MOLINA es Experto Universitario en Estadística Pública y en Métodos Avanzados de Estadística Aplicada. Ha trabajado durante dos años en la Unidad Estadística de la Consejería de Cultura, donde ha colaborado en varios proyectos. Desde 2005 trabaja en el Instituto de Estudios Sociales Avanzados (IESA-CSIC), donde realiza labores de apoyo estadístico a diversos proyectos, dedicándose principalmente a las líneas de investigación sobre nuevas tecnologías, turismo e inmigración.

\section{RECIBIDO: 15/01/10}

ACEPTADO:22/06/10

Publicado on-line: 04/03/2011 